\title{
Generation and spatio-temporal evolution of polarization speckle
}

Wang, Wei; Ma, Ning; Ritter, Jonas; Hanson, Steen Grüner; Osten, Wolfgang; Takeda, Mitsuo

\section{Published in:}

Proceedings of SPIE

Link to article, DOI:

$10.1117 / 12.2319920$

Publication date:

2018

Document Version

Publisher's PDF, also known as Version of record

Link back to DTU Orbit

Citation (APA):

Wang, W., Ma, N., Ritter, J., Hanson, S. G., Osten, W., \& Takeda, M. (2018). Generation and spatio-temporal evolution of polarization speckle. In Proceedings of SPIE (Vol. 10834). [108340K] SPIE - International Society for Optical Engineering. Proceedings of SPIE - The International Society for Optical Engineering https://doi.org/10.1117/12.2319920

\section{General rights}

Copyright and moral rights for the publications made accessible in the public portal are retained by the authors and/or other copyright owners and it is a condition of accessing publications that users recognise and abide by the legal requirements associated with these rights.

- Users may download and print one copy of any publication from the public portal for the purpose of private study or research.

- You may not further distribute the material or use it for any profit-making activity or commercial gain

- You may freely distribute the URL identifying the publication in the public portal 


\section{Generation and spatio-temporal evolution of polarization speckle}

Wei Wang, Ning Ma, Jonas Ritter, Steen G. Hanson, Wolfgang Osten, et al.

Wei Wang, Ning Ma, Jonas Ritter, Steen G. Hanson, Wolfgang Osten, Mitsuo Takeda, "Generation and spatio-temporal evolution of polarization speckle," Proc. SPIE 10834, Speckle 2018: VII International Conference on Speckle Metrology, 108340K (7 September 2018); doi: 10.1117/12.2319920

Event: SPECKLE 2018: VII International Conference on Speckle Metrology, 2018, Janów Podlaski, Poland 


\title{
Generation and Spatio-Temporal Evolution of Polarization Speckle
}

\author{
Wei Wang, ${ }^{\mathrm{a}, \mathrm{b}, *}$ Ning Ma, ${ }^{\mathrm{a}}$ Jonas Ritter, ${ }^{\mathrm{a}, \mathrm{c}}$ Steen G. Hanson, ${ }^{\mathrm{d}}$ Wolfgang Osten, ${ }^{\mathrm{c}}$ and Mitsuo Takeda ${ }^{\mathrm{b}, \mathrm{e}}$ \\ ${ }^{a}$ Institute of Photonics and Quantum Sciences, School of Engineering and Physical Science, \\ Heriot-Watt University, Edinburgh, United Kingdom, EH14 4AS; \\ ${ }^{\mathrm{b}}$ School of Optoelectronic Engineering, Xi'an Technological University, Xi'an 710032, China \\ ${ }^{\mathrm{c}}$ Institut für Technische Optik, Universität Stuttgart, Pfaffenwaldring 9, D-70569 Stuttgart, Germany \\ ${ }^{\mathrm{d}}$ DTU Fotonik, Department of Photonics Engineering, \\ Technical University of Denmark, Dk-4000 Roskilde, Denmark \\ ${ }^{\mathrm{e}}$ Center for Optical Research and Education (CORE), Utsunomiya University, 7-1-2 Yoto, \\ Utsunomiya, Tochigi 321-8585, Japan
}

\begin{abstract}
In this paper, we review our recent work on the statistical properties of polarization speckle generated by a birefringent material with rough surface. After a short introduction of a less-known concept of polarization speckle with its unique properties of random polarization states fluctuating in space, we provide an intuitive explanation of the cause of polarization speckle by vector random walks in the complex plane for two components of the vectorial electric fields. The surface polarization scattering is investigated in terms of the coherence matrix, and a relationship between the statistical properties of the scattered light at the scattering surface and the micro-structure of the anisotropic media has been explored to understand the underlying mechanism. The coherence and polarization properties of the stochastic electric fields at the far field after propagation have been studied in order to describe their spatial structure and evolution. Furthermore, the dynamic properties of polarization speckle have also been investigated in order to investigate the simultaneous reduction of coherence and polarization of the scattered light for the first time.
\end{abstract}

Keywords: Speckles, polarization, mutual coherence tensor, statistical optics, random walk

\section{INTRODUCTION}

Since continuous-wave lasers became commercially available in the early 1960s, extensive studies have been made on their basic properties and applications of laser speckle [1-3]. The term speckle patterns is usually associated with the fine-scale granular distribution of a light intensity pattern that arises from the interference of coherently superposed multiple random optical fields. In the majority of studies on speckle phenomena, these random optical fields have been treated as scalar optical fields, and the main interest has been in the statistical properties and applications of the intensity distribution of the speckle patterns. Recently, statistical properties of random electric vector fields have come to attract new interest because of their importance in wide areas of practical applications such as in biology and metrology. Statistical phenomena of random electric vector fields referred to as Polarization Speckle have relevance to the theories of speckles, polarization and coherence. Much effort is now being made by researchers to establish a new realm of statistical optics based on a unified theory on speckles, coherence and polarization [3,4]. Clearly it is far beyond the ability of the authors to cover these complete subjects related to the phenomena of stochastic electric fields. We will therefore restrict ourselves to the narrow-scope review on some of our recent works on statistical properties of polarization-related speckle phenomena, along with an introduction of mechanism for generation of polarization speckles, their coherence and polarization properties, spatial structure and evolution. Furthermore, we will also introduce some dynamic properties of polarization speckle produced by a rotating rough-surfaced retardation plate to explore the simultaneous reduction of coherence and polarization of the scattered light.

*w.wang@hw.ac.uk Tel: +44 (0) 131451 3141; Fax: +44 (0) 1314513129 


\section{INTUITIVE EXPLANATION OF THE CAUSE OF POLARIZATION SPECKLE}

Similar to the conventional laser speckle composed of a multitude of independently phased additive complex components, polarization speckle known as stochastic electric fields is a vectorial signal, which could be understood as a sum of many contributions from polarization phasors with random vector directions (random polarization states) and random complex amplitudes (random amplitudes and random phases) representing a monochromatic or nearly monochromatic vectorial electric field disturbance. For each component of polarization speckle, a complex addition of many small independent contributions from the scalar components of the polarization phasors constitutes a random walk in the complex plane and the resultant phasor of the sum establishes the complex amplitude. Figure 1 illustrates an example of polarization phasor sum based on the vector random walks for $\tilde{E}_{x}$ and $\tilde{E}_{y}$, respectively. Given that the complex electric field of the random walk can be expressed as [5]

$$
\mathbf{E}=\tilde{E}_{x} \hat{x}+\tilde{E}_{y} \hat{y}=a_{x} e^{i \phi_{x}} \hat{x}+a_{y} e^{i \phi_{y}} \hat{y}=\frac{1}{\sqrt{N}} \sum_{n=1}^{N} \mathbf{E}_{n}=\frac{1}{\sqrt{N}} \sum_{n=1}^{N}\left(a_{x n} e^{i \phi_{x n}} \hat{x}+a_{y n} e^{i \phi_{y n}} \hat{y}\right),
$$

where $\mathbf{E}$ represents the resultant polarization phasor (a complex electric field vector), $\tilde{E}_{x}$ and $\tilde{E}_{y}$ are two Cartesian components of $\mathbf{E}$ along the $\hat{x}$ and $\hat{y}$ directions, with them being unit vectors. $a_{x}$ and $a_{y}$ are the lengths and $\phi_{x}$ and $\phi_{y}$ are the phases of the resultant polarization components, respectively. $N$ represents the number of polarization phasor components in the random walk, $\mathbf{E}_{n}$ represents the $n$-th component of the polarization phasor in the sum (a complex vector), $\quad a_{k n}$ and $\phi_{k n}$ are the length and phase for $k=x$ or $y$ components of $\vec{E}_{n}$ respectively. The scaling factor $1 / \sqrt{N}$ is introduced here in order to preserve finite energy, i.e. $\overline{|\mathbf{E}|^{2}}$, even when the number of component polarization phasors approach infinity.
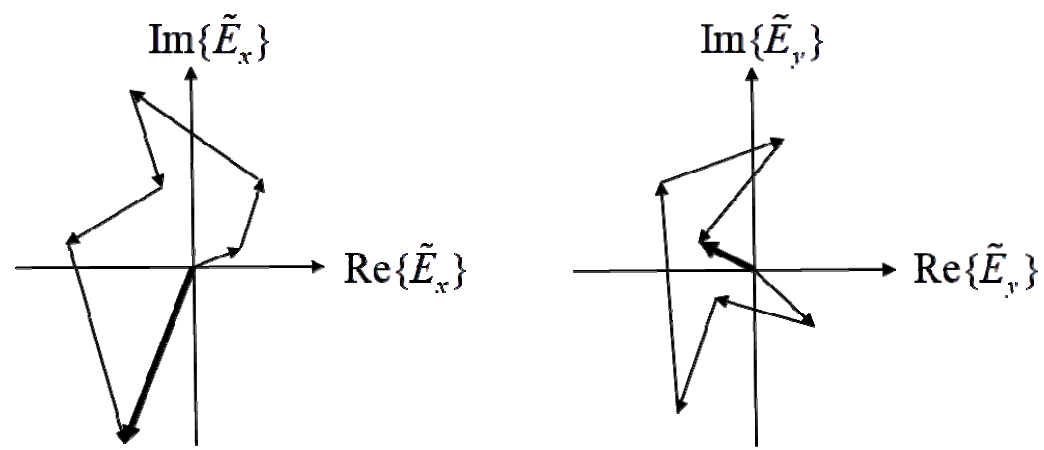

Figure 1 Schematic diagram of random polarization phasor sum

When these components of the polarization phasors are added vectorially together, they constitute what is known as a "vectorial random walk." The sums for each polarization components may be large or small, depending on the relative phases of the various components of the sum, and in particular whether constructive or destructive interference dominates the sum, and therefore the polarization state will be determined from the resultants of sums for these two polarization components of the vectorial electric field.

\section{DEPENDENCE OF PLARIZATON SPECKLE ON MICROSTRUCTURE OF A BIREFRINGENT MATERIAL}

In this section, we will review our recent work on the generation of polarization speckle based on surface scattering of electric fields, and explore how various statistical properties polarization speckle depend on the microstructure of the rough surface of birefringent materials [6,7].

As shown in Fig. 2, we assume free-space transmission geometry for surface polarization scattering, where an example of a cross-section of the birefringent material with a typical random surface height fluctuation has been given. Just as the laser speckle generated from the rough surface, the relationship between these height variations of the rough- 
surfaced retardation plate and the amplitude variations of the scattered electric field is in general extremely complicated. It is influenced by variations of surface slope, shadowing of retardation plate, multiple scattering and reflection inside the birefringent material, and polarization effects introduced to the scattered wave travelling in the retardation plate in arbitrary directions with reference to the orientation of fast/slow axis of the birefringent material. For analysis purpose, in this letter we will adopt an oversimplified model to give some physical insight into the relationship between the surface height fluctuations for the rough-surfaced retardation plate and the polarization fluctuations of the scattered electric field.

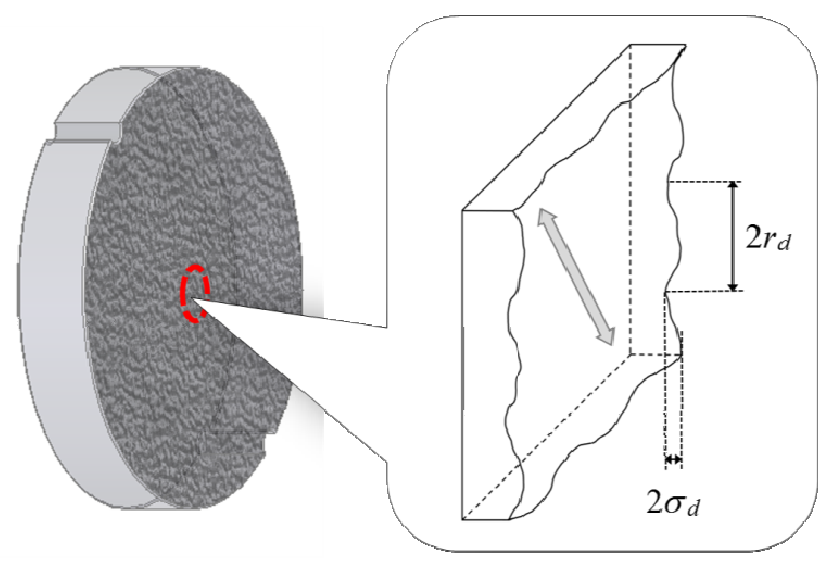

Figure 2 Schematic diagram of surface scattering from a rough surface of anisotropic media with an example of a crosssection of a typical surface thickness fluctuation

Let the scattered electric field immediately behind the rough surface, $\mathbf{E}^{t}(\mathbf{r})$, be related to the incident electric vector $\mathbf{E}^{i}(\mathbf{r})$ through the Jones matrix $\mathbf{T}(\mathbf{r})$ for a birefringent material with its fast/slow axis aligned along the $\hat{y}$ direction given by

$$
\mathbf{T}(\mathbf{r})=\left(\begin{array}{cc}
e^{-j \phi_{x}(\mathbf{r})} & 0 \\
0 & e^{-j \phi_{y}(\mathbf{r})}
\end{array}\right)
$$

where $\phi_{m}(m=x, y)$ is the effective phase delay for the $\hat{x}$ or $\hat{y}$ components of the electric field introduced by an optical path passing through the retardation plate with surface height fluctuation and the remaining region being free space. Under the assumption that the coherence property of the incident illumination field and the correlation property of the depolarizer are statistically independent, we have the transmission coherence matrix $\boldsymbol{W}^{t}\left(\mathbf{r}_{1}, \mathbf{r}_{2}\right)$ of the modulated fields just behind the depolarizer. That is

$$
\boldsymbol{W}^{t}\left(\mathbf{r}_{1}, \mathbf{r}_{2}\right)=\left(\begin{array}{ll}
W_{x x}^{i}\left(\mathbf{r}_{1}, \mathbf{r}_{2}\right)<e^{j \Delta \phi_{x x}\left(\mathbf{r}_{1}, \mathbf{r}_{2}\right)}> & W_{x y}^{i}\left(\mathbf{r}_{1}, \mathbf{r}_{2}\right)<e^{j \Delta \phi_{y y}\left(\mathbf{r}_{1}, \mathbf{r}_{2}\right)}> \\
W_{y x}^{i}\left(\mathbf{r}_{1}, \mathbf{r}_{2}\right)<e^{j \Delta \phi_{y x}\left(\mathbf{r}_{1}, \mathbf{r}_{2}\right)}>W_{y y}^{i}\left(\mathbf{r}_{1}, \mathbf{r}_{2}\right)<e^{j \Delta \phi_{y y}\left(\mathbf{r}_{1}, \mathbf{r}_{2}\right)}>
\end{array}\right),
$$

with the angular brackets $\langle\cdots\rangle$ above denoting "ensemble average". For demonstration purposes only, and without loss of generality, we assume that the incident light is a linearly polarized, spatially coherent, plane wave for which the coherence matrix is given by

$$
\boldsymbol{W}^{i}\left(\mathbf{r}_{1}, \mathbf{r}_{2}\right)=I_{0}\left(\begin{array}{cc}
\cos ^{2} \theta & \cos \theta \sin \theta \\
\cos \theta \sin \theta & \sin ^{2} \theta
\end{array}\right)
$$


where $I_{0}$ is the on-axis intensity of the incident field and $\theta$ is the linear polarization angle with the $\hat{x}$-axis. Some further progress can be made for Eq. (3) when certain assumptions for the effective phase delay and the correlation function of the surface thickness are specified [2-3]. For simplicity, the assumption is usually made that the effective phase delays (or equivalently the surface height fluctuations) is a Gaussian random process. We have

$$
\begin{aligned}
<e^{j \Delta \varphi_{\text {Im }}}>= & \left\langle\exp \left\{j(2 \pi / \lambda)\left[\left(n_{l}-1\right)\left(\bar{d}+d\left(\mathbf{r}_{1}\right)\right)-\left(n_{m}-1\right)\left(\bar{d}+d\left(\mathbf{r}_{1}\right)\right)\right]\right\}\right\rangle \\
= & \exp \left\{(j 2 \pi / \lambda)\left(n_{l}-n_{m}\right) \bar{d}\right\}\left\langle\exp \left\{j(2 \pi / \lambda)\left[\left(n_{l}-1\right) d\left(\mathbf{r}_{1}\right)-\left(n_{m}-1\right) d\left(\mathbf{r}_{2}\right)\right]\right\}\right\rangle \\
= & \exp \left\{(j 2 \pi / \lambda)\left(n_{l}-n_{m}\right) \bar{d}\right\} \exp \left\{-\left(2 \pi^{2} / \lambda^{2}\right)\left[\left(n_{l}-1\right)^{2}<d^{2}\left(\mathbf{r}_{1}\right)>\right.\right. \\
& \left.\left.+\left(n_{m}-1\right)^{2}<d^{2}\left(\mathbf{r}_{2}\right)>-2\left(n_{l}-1\right)\left(n_{m}-1\right)<d\left(\mathbf{r}_{1}\right) d\left(\mathbf{r}_{2}\right)>\right]\right\},
\end{aligned}
$$

where $\lambda$ is the wavelength in vacuum, $d(r)$ is the zero mean Gaussian random thickness variation around the average thickness $\bar{d}$ of the birefringent plate, $n_{l}$ and $n_{m}$ are the refractive indices for the birefringent material. Another assumption is that the correlation function of the surface thickness also takes Gaussian form.

$$
<d\left(\mathbf{r}_{1}\right) d\left(\mathbf{r}_{2}\right)>=\sigma_{d}^{2} \exp \left\{-\left|\mathbf{r}_{1}-\mathbf{r}_{2}\right|^{2} / r_{d}^{2}\right\},
$$

where $\sigma_{d}^{2}$ and $r_{d}$ are mutually independent quantities indicating the variance of $d(r)$ and the radius at which the normalized surface thickness correlation falls to $1 / e$, respectively. Therefore, the transmission coherence tensor of the scattered electric field immediately behind the scattering layer becomes a complex Hermitian matrix.

$$
\begin{aligned}
& W_{x x}^{t}(\Delta r)=I_{0} \cos ^{2} \theta \exp \left\{-4 \pi^{2}\left(\sigma_{d} / \lambda\right)^{2}\left(n_{x}-1\right)^{2}\left[1-\exp \left(-\Delta r^{2} / r_{d}^{2}\right)\right]\right\}, \\
& W_{y y}^{t}(\Delta r)=I_{0} \sin ^{2} \theta \exp \left\{-4 \pi^{2}\left(\sigma_{d} / \lambda\right)^{2}\left(n_{y}-1\right)^{2}\left[1-\exp \left(-\Delta r^{2} / r_{d}^{2}\right)\right]\right\}, \\
W_{x y}^{t}(\Delta r)= & {\left[W_{y x}^{t}(\Delta r)\right]^{*}=I_{0} \sin \theta \cos \theta \exp \left\{j 2 \pi \bar{d}\left(n_{x}-n_{y}\right) / \lambda\right\} \exp \left\{-2 \pi^{2}\left(\sigma_{d} / \lambda\right)^{2}\left[\left(n_{x}-1\right)^{2}+\left(n_{y}-1\right)^{2}\right.\right.} \\
& \left.\left.-2\left(n_{x}-1\right)\left(n_{y}-1\right) \exp \left(-\Delta r^{2} / r_{d}^{2}\right)\right]\right\} .
\end{aligned}
$$

When Equations (7.1)-(7.3) have been derived, the surface of the polarization scattering spot is assumed to be rough and wide-sense stationary where its correlation function depends only on the differences of measurement coordinates: $\Delta r=\left|\mathbf{r}_{1}-\mathbf{r}_{2}\right|$. These results provide us with a specific relationship between the correlation properties of the transmitted electric fields and the micro-structure of the rough surface of the birefringent material.

To provide physical insight into these results, we have presented some numerical examples by taking the following parameters: $\theta=\pi / 4, n_{x}=1.486$ and $n_{y}=1.658$ for the birefringent material: calcite [8]. Note the fact that the normalized correlation functions in Eqs. (7) approach their non-zero asymptotes, respectively for large separation $\Delta r$. These asymptotes indicate that the transmitted electric field passing through the retardation plate has a non-negligible specular transmission of the incident light, as can be easily seen by noting the flat correlation functions when $\sigma_{d}=0$, in which only a specular transmission light exists. To study the non-specular component in the scattered light, it is helpful to subtract out these asymptotes of the correlation functions, yielding

$$
' W_{m n}^{t}(\Delta r)=W_{m n}^{t}(\Delta r)-W_{m n}^{t}(+\infty) .
$$

The coherence area $A_{c}$ of this non-specular component of the scattered electric field is found by evaluating [2]

$$
A_{c}=2 \pi \int_{0}^{+\infty}\left(\mu^{t}\right)^{2} \Delta r d \Delta r
$$

where 


$$
\left(\mu^{t}\right)^{2}=\frac{\left|W_{x x}^{t}(\Delta r)\right|^{2}+\left|' W_{x y}^{t}(\Delta r)\right|^{2}+\left|{ }^{\prime} W_{y x}^{t}(\Delta r)\right|^{2}+\left|W_{y y}^{t}(\Delta r)\right|^{2}}{\left(\left|' W_{x x}^{t}(0)\right|+\left|W_{y y}^{t}(0)\right|\right)^{2}} .
$$

The coherence area $A_{c}$ in Eq. (9) provides a useful measure of the average "size" of the scattered electric field. It is readily seen that when only a specular transmission exists with $\sigma_{d}=0,\left(\mu^{t}\right)^{2}$ for the scattered electric field becomes: $\lim _{\sigma_{d} / \lambda \rightarrow 0}\left(\mu^{t}\right)^{2}=e^{-2\left(\Delta r / r_{d}\right)^{2}}$ and the corresponding surface thickness correlation area can be estimated as $A_{s t c}=\pi r_{d}^{2} / 2$.

Another issue here is the static random spatial distribution of the polarization state for the scattered light. To specify the statistical property for surface polarization scattering, we will use the spatial degree of polarization defined by $[9,10]$

$$
P(\mathbf{r})=\left\{1-\frac{4 \operatorname{det} \boldsymbol{W}(\mathbf{r}, \mathbf{r})}{[\operatorname{tr} \boldsymbol{W}(\mathbf{r}, \mathbf{r})]^{2}}\right\}^{1 / 2},
$$

where $t r$ and det indicate the trace and determinant of the matrix, respectively. Here the spatial average has been adopted for ensemble average when the coherence matrix in Eq. (3) and Eqs. (7) have been estimated. On substituting from Eqs. (7.1)-(7.3) into Eq. (11), we obtain the spatial degree of polarization for the scattered light immediately behind the depolarizer surface. That is $P^{t}=\sqrt{1-\sin ^{2}(2 \theta)\left[1-\exp \left\{-k^{2} \sigma_{d}^{2}\left(n_{x}-n_{y}\right)^{2}\right\}\right]}$. Similar to the coherence analysis above, to study the stochastic polarization property for the non-specular component of the scattered light, the spatial degree of polarization can also be estimated by substituting Eq. (8) for $\Delta r=0$ into Eq. (11) when the asymptotes of the correlation functions for the element in the coherence matrix have been subtracted.

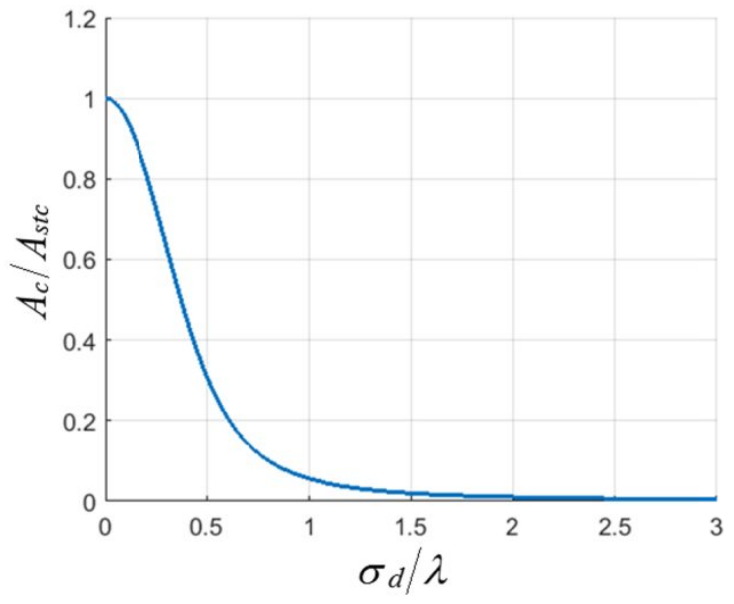

(a)

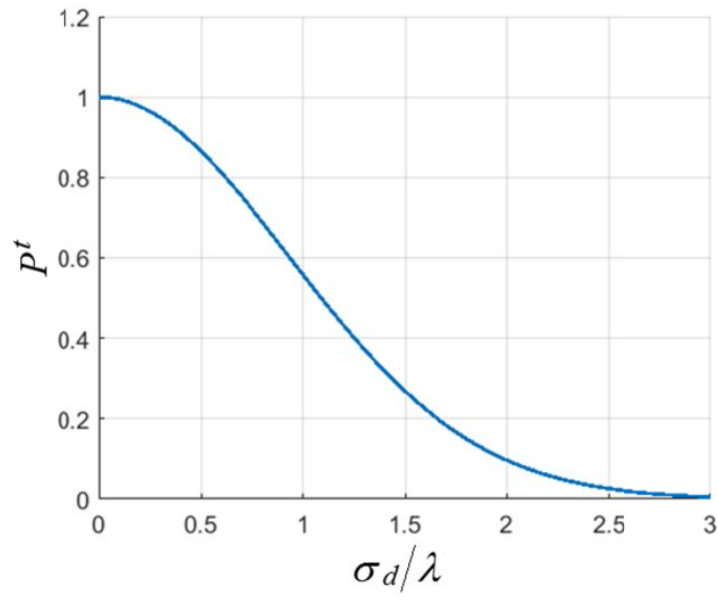

(b)

Figure 3 Normalized coherence area $A_{c} / A_{s t c}$ vs. $\sigma_{d} / \lambda$, (a); Spatial degree of polarization for polarization speckle vs. $\sigma_{d} / \lambda$, (b).

Figure 3(a) shows a plot of the scattered field correlation area $A_{c}$ normalized by the surface-thickness correlation area $A_{s t c}$ vs the standard deviation of the rough surface fluctuation normalized by the incident wavelength $\sigma_{d} / \lambda$. Note that when the standard deviation of the rough-surface fluctuation approaches the wavelength of the incident wave, the correlation area of the scattered electric fields is about $1 / 20$ of the surface-thickness correlation area. The reason for the shrinking of the correlation area for the scattered field, as the standard deviation of the surface roughness increases, lies in the fact that when the surface thickness fluctuation for the retardation plate begin to exceed the incident wavelength, the phase difference between the $\hat{x}$ and $\hat{y}$ components of the electric fields is beyond $2 \pi$ and the scattered field coming from such rough-surfaced retardation plate will contain rays with all states of polarization. Clearly, for large value of $\sigma_{d}$, the correlation area of the scattered electric field is much smaller than the correlation area of the surface itself. 
Figure 3(b) shows the spatial degree of polarization for the scattered electric field vs. the standard deviation for the surface thickness fluctuation normalized by the wavelength. As $\sigma_{d} / \lambda$ increases, the spatial degree of polarization reduces to zero monotonically. Note that when the surface-thickness standard deviation reaches beyond $2 \lambda$, the spatial degree of polarization for the scattered light at the surface is less than $1 / 10$ indicating a depolarization effect to scramble the incident polarization. Therefore, the scattered electric field at the surface can realistically be considered as spatially unpolarized light. Comparing Fig. 3(a) and Fig. 3(b), we further note that the widths at half maximum of $P^{t}$ is much broader than that of $A_{c} / A_{s t c}$ indicating that the realization of depolarization is much more difficult than that of decoherence for the scattered light introduced by increasing the standard deviation of the rough-surfaced retardation plate.

Under the assumption of a large surface roughness with large phase variance owing to the smaller lateral distance required to obtain a phase difference of $2 \pi$, we can make further progress. In this case the second exponential terms in Eq. (7) may be series expanded, and only the first two terms in the series are essential. For $\left(n_{l}-1\right)\left(n_{m}-1\right) k^{2} \sigma_{d}{ }^{2}>>(2 \pi)^{2}$, $(l, m=x, y)$, we have

$$
\left\langle\exp \left\{j \Delta \varphi_{l m}\right\}\right\rangle=\exp \left\{-k^{2} \sigma_{d}^{2} / 2\left[\left(n_{l}-n_{m}\right)^{2}+2\left(n_{l}-1\right)\left(n_{m}-1\right)|\Delta \mathbf{r}|^{2} / r_{d}^{2}\right]\right\} .
$$

The approximation in Eq. (12) has resulted in breaking down the double exponential into a single exponential facilitating further mathematical analysis in order later to arrive at an analytical solutions for the propagation of the coherence matrix. Under the paraxial approximation, the propagation of the coherence matrix through a complex-valued ABCD optical system is given by

$$
\mathbf{W}^{o}\left(\mathbf{p}_{1}, \mathbf{p}_{2}\right)=\iint \mathbf{W}^{t}\left(\mathbf{r}_{1}, \mathbf{r}_{2}\right) G^{*}\left(\mathbf{r}_{1}, \mathbf{p}_{1}\right) G\left(\mathbf{r}_{2}, \mathbf{p}_{2}\right) d \mathbf{r}_{1} d \mathbf{r}_{2} .
$$

where the superscript ${ }^{O}$ indicates the field in the observation plane, and the Green's function in the matrix formalism is

$$
G(\mathbf{r}, \mathbf{p})=-\frac{j k}{2 \pi B} \exp \left\{-\frac{j k}{2 B}\left(A|\mathbf{r}|^{2}-2 \mathbf{r} \cdot \mathbf{p}+D|\mathbf{p}|^{2}\right)\right\} .
$$

In the equation above, $j$ is the imaginary unit and the $A, B$ and $D$ are complex values that can be determined by multiplying the matrices for all the individual optical components in the optical train, i.e. lenses, free space propagation and apertures [11-12]. The benefit of the above expression in Eq. (6) is that it usually enables us to give an analytical result, covering a broad range of optical systems. In arriving at the above expression, we have tacitly assumed that the refractive indices in the input and output planes are identical, but not necessarily unity. As an example, we will consider a typical case of free space propagation over a distance $z$ with a preceding aperture of size $r_{s}$ to model the illuminating spot size, and thus the corresponding $\mathrm{ABCD}$ matrix is

$$
\left(\begin{array}{cc}
1-j z / z_{R} & z \\
-j / z_{R} & 1
\end{array}\right)
$$

where $z_{R}$ is the Rayleigh range $z_{R}=k r_{s}^{2} / 2$. By substituting these elements of the ABCD matrix into Eq. (14), one can study the changes in the degree of coherence and the degree of polarization for free space propagation.

Figure 4(a) gives the absolute values of the degree of coherence of the polarization speckle generated by the roughsurfaced retardation plate at two positions $\Delta \mathbf{p}=\mathbf{p}_{1}-\mathbf{p}_{2}$ located symmetrically with respect to the $\hat{z}$ axis along the normalized propagation distance. It can readily be seen that the degree of coherence takes a large value close to unity for two points located near the optical axis. Fig. 4(b) shows the degree of polarization of the polarization speckle for freespace propagation, plotted against the normalized propagation distance $z / z_{R}$, and the normalized lateral distance measured in spot size $p / r_{s}$. As expected, the degree of polarization changes appreciably depending both on the propagation distance $z$ and on the polarization angle of the incident beam. Instead of a uniform distribution for the modulated electric fields just behind the depolarizer, the spatial degree of polarization for the polarization speckle after propagation does not remain uniform. 


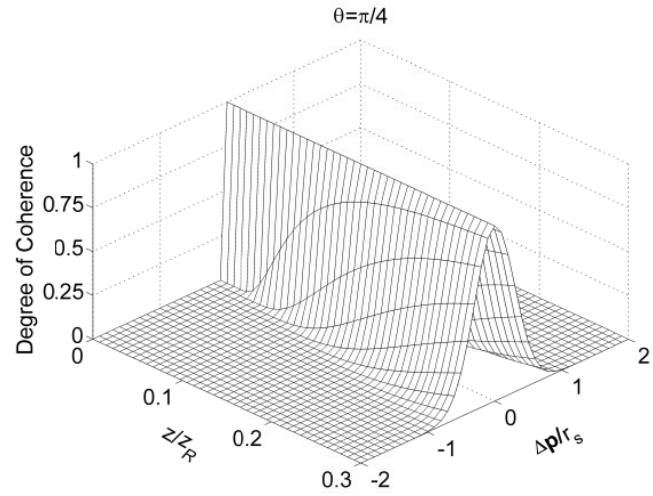

(a)

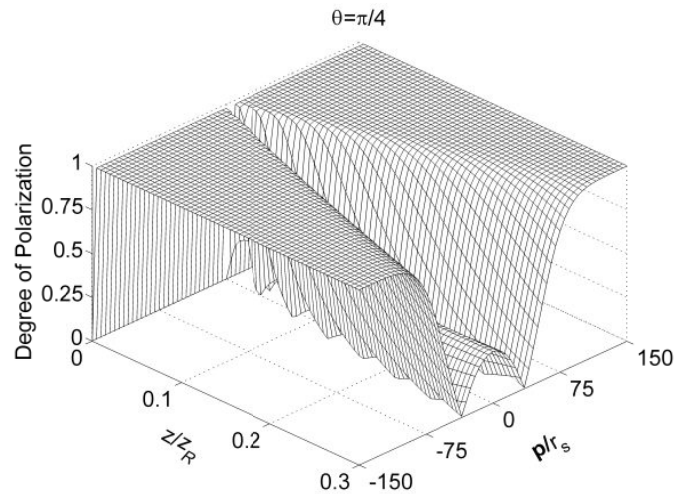

(b)

Figure 4 Degree of Coherence for free-space propagation at two points located symmetrically (a); Degree of polarization for free-space propagation as a function of the lateral distance measured in spot size units and the propagation distance measured in units of the Rayleigh length (b).

\section{THREE-DIMENSIONAL STRUCTURE OF POLARIZATION SPECKLE}

Until now, we have considered only the propagation properties of polarization speckle in a measurement plane parallel to the scattering surface. We now turn our attention to the 3-dimensional structure of polarization speckle with both transverse and longitudinal properties. The statistical properties of polarization speckle in depth are those along the direction normal to the scattering plane. In particular, we wish to find the distance in depth over which a speckle pattern remains correlated, just as we found the transverse correlation width in previous section. While, both correlation width and correlation depth will vary, depending on the particular coordinates chosen, nonetheless the results are representative of the results elsewhere from a statistical point of view. Therefore, we will calculate the coherence tensor with its each element of cross-correlation for the observed fields at two different positions, which we represent by $W_{m n}^{o}\left(\rho_{1}, z ; \rho_{2}, z+\Delta z\right)=<\tilde{E}_{m}\left(\rho_{1}, z\right) \tilde{E}_{n}\left(\rho_{2}, z+\Delta z\right)>$.

From Equations (3), (7) and (12), the mutual coherence tensor for the scattered electric fields just behind the rough-surfaced retardation plate can be approximately rewritten as

$$
W_{l m}^{t}\left(\mathbf{r}_{1}, \mathbf{r}_{2}\right) \approx W_{l m}^{i}\left(\mathbf{r}_{1}, \mathbf{r}_{2}\right) \frac{\pi r_{d}^{2} \delta(\Delta \mathbf{r})}{k^{2} \sigma_{d}^{2}\left(n_{l}-1\right)\left(n_{m}-1\right)} \exp \left\{-\frac{k^{2} \sigma_{d}^{2}\left(n_{l}-n_{m}\right)^{2}}{2}\right\},
$$

When Eq. (16) is derived, we have made use of the Dirac delta correlation assumption based on the fact that $\lim _{\alpha \rightarrow 0} \exp \left\{-|\Delta \mathbf{r}|^{2} / \alpha^{2}\right\}=\pi \alpha^{2} \delta(\Delta \mathbf{r})$. Such assumption will facilitate further mathematical analysis in order to arrive at analytical solutions for the propagation of the coherence tensor through an ABCD optical system as shown in Fig. 5.

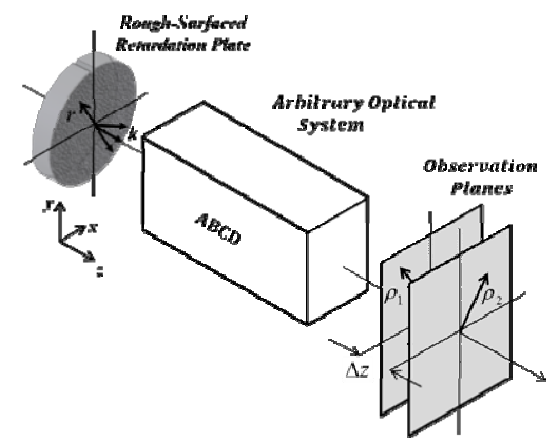

Figure 5 Schematic for study the 3-D structure of polarization speckle propagation through a complex ABCD optical system 
Under the paraxial approximation, the mutual coherence matrix for the electric field at $\boldsymbol{\rho}_{1}$ on $1^{\text {st }}$ observation plane after passing through a complex ABCD optical system and that for the field at $\boldsymbol{\rho}_{2}$ on $2^{\text {nd }}$ plane displaced by a distance $\Delta z$ is connected by Eq. (13) and (14). Let $\mathbf{M}_{1}$ be ABCD matrix for the entire optical system under consideration, determined by the multiplication of the matrices for all the individual optical components, i.e., the lenses, free space propagations and aperture. For our system in Fig. 5, the relationship between $\mathrm{ABCD}$ matrix $\mathbf{M}_{1}$ at the $1^{\text {st }}$ observation plane and $\mathbf{M}_{2}$ for the $2^{\text {nd }}$ observation plane can be chained together as:

$$
\mathbf{M}_{2}=\left(\begin{array}{cc}
1 & \Delta z \\
0 & 1
\end{array}\right) \mathbf{M}_{1} .
$$

On substituting from Eq. (17) into Eq. (13), we obtain the expressions for the propagation of coherence tensor for the polarization speckle at two observation planes with a separation distance of $\Delta z$. That is

$$
\mathbf{W}^{o}\left(\boldsymbol{\rho}_{1}, \boldsymbol{\rho}_{2}, \Delta z\right)=\frac{I_{o} r_{d}^{2} \sin \theta \cos \theta \pi}{k^{2} \sigma_{d}^{2}\left(n_{x}-1\right)\left(n_{y}-1\right)} W\left(\boldsymbol{\rho}_{1}, \boldsymbol{\rho}_{2}, \Delta z\right)\left(\begin{array}{cc}
\frac{\cot \theta\left(n_{y}-1\right)}{\left(n_{x}-1\right)} & \exp \left\{-\frac{k^{2} \sigma_{d}{ }^{2}\left(n_{x}-n_{y}\right)^{2}}{2}\right\} \\
\exp \left\{-\frac{k^{2} \sigma_{d}^{2}\left(n_{x}-n_{y}\right)^{2}}{2}\right\} & \frac{\tan \theta\left(n_{x}-1\right)}{\left(n_{y}-1\right)}
\end{array}\right),
$$

where

$$
\begin{aligned}
W\left(\boldsymbol{\rho}_{1}, \boldsymbol{\rho}_{2}, \Delta z\right)= & \frac{j k}{2 \pi\left[-\Delta z\left(C B^{*}-D A^{*}\right)+2 j \operatorname{Im}\left(B A^{*}\right)\right]} \exp \left\{-\frac{j k}{2}\left[-\left|\boldsymbol{\rho}_{1}\right|^{2}\left(\frac{D}{B}\right)^{*}+\left|\boldsymbol{\rho}_{2}\right|^{2} \frac{D}{B+D \Delta z}\right]\right\} \\
& \times \exp \left\{-\frac{j k\left[-2 \boldsymbol{\rho}_{1} \cdot \boldsymbol{\rho}_{2}\left(B^{*} B+B^{*} D \Delta z\right)+\left|\boldsymbol{\rho}_{2}\right|^{2}\left(B^{*}\right)^{2}+\left|\boldsymbol{\rho}_{1}\right|^{2}(B+D \Delta z)^{2}\right]}{2 B^{*}(B+D \Delta z)\left[-\Delta z\left(C B^{*}-D A^{*}\right)+2 j \operatorname{Im}\left(B A^{*}\right)\right]}\right\} .
\end{aligned}
$$

Without loss of generality and for demonstration purposes only, the optical system has been chosen as free-space propagation with its ABCD matrix given in Eq. (15). From the analytic expressions of the coherence tensor in Eq. (18) and (19), we can obtain the 3-D distribution for the degree of coherence and degree of polarization for polarization speckle. They are

$$
\begin{aligned}
\mu\left(\boldsymbol{\rho}_{1}, \boldsymbol{\rho}_{2}, \Delta z\right)= & {\left[1-\left(\Delta z z_{R} j\right) /[2(\Delta z+z) z]\right]^{-1} \exp \left\{-\frac{j k}{2}\left[\left|\boldsymbol{\rho}_{1}\right|^{2} / z-\left|\boldsymbol{\rho}_{2}\right|^{2} /(\Delta z+z)\right]\right\} } \\
& \times \exp \left\{-k z_{R}\left|\boldsymbol{\rho}_{2} /(\Delta z+z)-\boldsymbol{\rho}_{1} / z\right|^{2} / 2\left[2-\Delta z z_{R} j /[(\Delta z+z) z]\right]\right\},
\end{aligned}
$$

and

$$
P(\boldsymbol{\rho})=\left\{1-4 \tan ^{2} \theta\left[1-\exp \left\{-k^{2} \sigma_{d}^{2}\left(n_{x}-n_{y}\right)^{2}\right\}\right]\left[\left(n_{y}-1\right) /\left(n_{x}-1\right)+\tan ^{2} \theta\left(n_{x}-1\right) /\left(n_{y}-1\right)\right]^{-2}\right\}^{1 / 2} .
$$

It's interesting to note that the spatial degree of polarization for the polarization speckle is independent of the location and stay constant during propagation. Figure 6 shows the contours of the 3-D correlation function with $\Delta \rho=\left|\rho_{1}-\rho_{2}\right|$. Note the fact that the illumination spot size $r_{s}$ is small compared with the distance to the observation plane $z$, and the transverse and longitudinal correlation widths have been measured by the widths of the curves at their half-maxima, i.e. $|\mu|=0.5$. From Fig. 6 , we find that a polarization speckle has a typical needle shaped structure and its extent in the axial dimension is much greater than the extent in the transverse dimension. Within this needle shaped structure, the polarization states have become scrambled and the perfectly polarized coherent incident beam has become spatially partially polarized or depolarized depending on the polarization direction of the incident electric field vector as compared to the optical axis of the birefringent material with rough surface. 


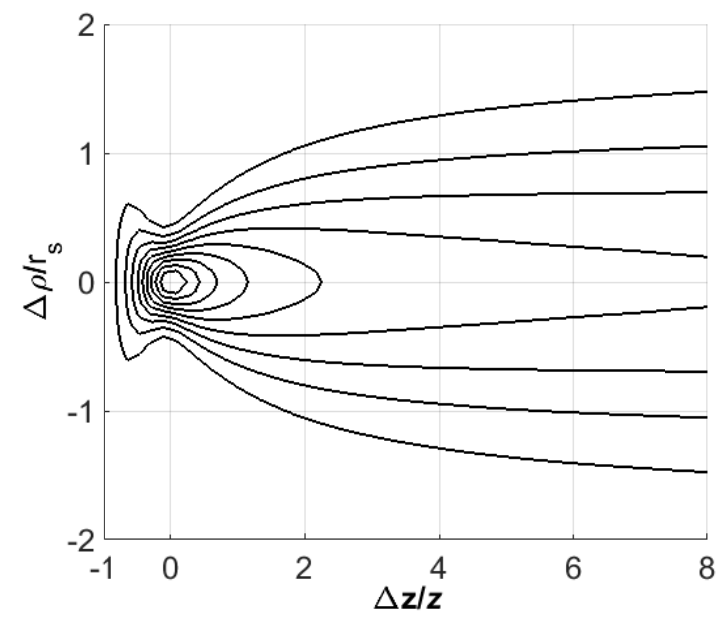

Fig. 6 Contours for 3-D correlation function $|\mu(\Delta \rho, \Delta z)|$.

\section{DYNAMIC POLARIZATION SPECKLE FOR REDUCTION OF COHERENCE AND POLARIZATION}

In the previous sections, we have discussed the statistical properties of the static polarization speckle. In some applications, such as polarization imaging with coherent light, the effects of polarization speckle are detrimental and become a distinct annoyance, and therefore ways should be sought to reduce or eliminate it through a reduction of temporal and spatial coherence and polarization. In this section, we will introduce a new concept proposed recently to suppress the polarization speckle for simultaneous reduction of coherence and polarization. Thus, the goal of this study is to discuss the statistics of dynamic polarization speckle and the corresponding effect of reduction of coherence and polarization, which damages the process of information extraction made by coherent light.

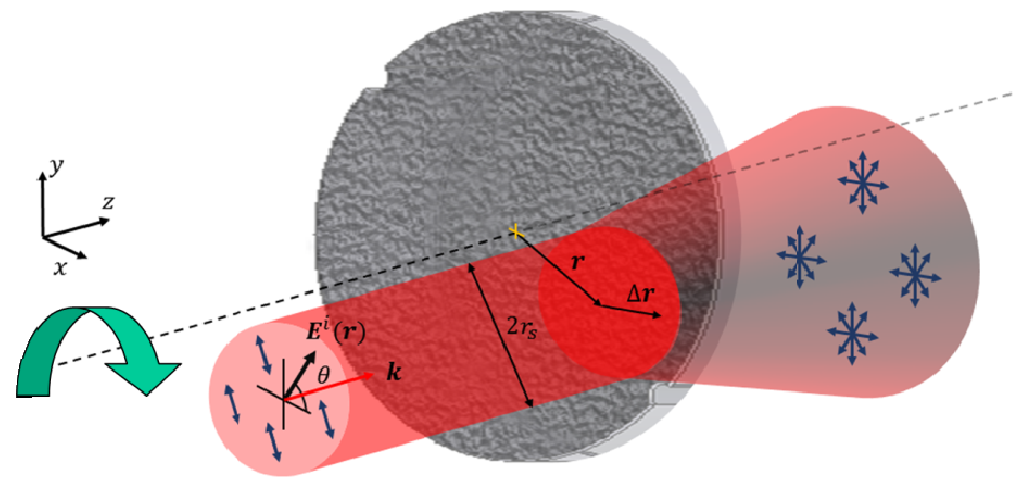

Figure 7 Simultaneous reduction of coherence and polarization by a rotating rough-surface retardation plate

Figure 7 is the schematic diagram of a rotating birefringent plate with a rough surface. As the depolarizer is rotated, any point behind the rough surfaced retardation plate experiences a changing phase delay between the $\hat{x}$ and $\hat{y}$ components of the incident light and generates a changing random walk of polarization phasor with its contribution changing with time in a complex way. In effect, new realizations of the vectorial random walk are created as time progresses. As a consequence, the polarization sates at any point changes with time. The detected intensity and polarization states measured with an integration time are simply the integrals over a number of independent polarization speckle realizations. Here we begin our detailed analysis and discussion from some fundamental concepts of polarization and coherence of stochastic light with its central concepts of mutual coherence tensor whose elements are mutual coherence function of each components of the electric fields, i.e., $W_{m n}\left(\mathbf{r}_{1}, \mathbf{r}_{2}, \tau\right)=<\tilde{E}_{m}^{*}\left(\mathbf{r}_{1}, t\right) \tilde{E}_{n}\left(\mathbf{r}_{2}, t+\tau\right)>$ with $\tau$ being a 
relative time delay. Let the fast/slow axis of the rough-surfaced birefringent plate be rotated to the angle $\theta(t)$ to $\hat{x}$ axis at the instant time $t$ and let its rotational angular velocity be $\omega$. Therefore, the Jones vector for the instantaneously transmitted electric field just behind the rough-surfaced birefringent plate becomes:

$$
\left(\begin{array}{c}
\tilde{E}_{x}^{o}(\mathbf{r}, t) \\
\tilde{E}_{y}^{o}(\mathbf{r}, t)
\end{array}\right)=\left(\begin{array}{cc}
\cos \theta(t) & -\sin \theta(t) \\
\sin \theta(t) & \cos \theta(t)
\end{array}\right)\left(\begin{array}{cc}
e^{-j \varphi_{x}(\mathbf{r}, t)} & 0 \\
0 & e^{-j \varphi_{y}(\mathbf{r}, t)}
\end{array}\right)\left(\begin{array}{cc}
\cos \theta(t) & \sin \theta(t) \\
-\sin \theta(t) & \cos \theta(t)
\end{array}\right)\left(\begin{array}{c}
\tilde{E}_{x}^{i}(\mathbf{r}, t) \\
\tilde{E}_{y}^{i}(\mathbf{r}, t)
\end{array}\right),
$$

where $\theta=|\omega| t+\theta_{0}$ with $\theta_{0}$ being the initial orientation angle for the fast/slow axis.

Our approach to finding the dynamic properties of polarization speckle and its dependence on depolarizer rotation will be to find the coherence time and coherence area through the mutual coherence tensor. In statistical optics, it's sometimes convenient to evaluate infinite time average by means of an ensemble average. Assuming that the rough-surfaced retardation plate is a stationary and ergodic Gaussian random process, we can evaluate the time average in a similar way as shown in Eq. (5). Further progress requires the adoption of a specific form for correlation of surface thickness. As before, we choose a Gaussian shape for this correlation function

$$
<d\left(\mathbf{r}_{1}, t\right) d\left(\mathbf{r}_{2}, t+\tau\right)>=\sigma_{d}^{2} \exp \left\{-|\Delta \mathbf{r}+\mathbf{r} \times \omega \tau|^{2} / r_{d}^{2}\right\},
$$

where $\mathbf{r}=\left(\mathbf{r}_{1}+\mathbf{r}_{2}\right) / 2$ and $\Delta \mathbf{r}=\left(\mathbf{r}_{1}-\mathbf{r}_{2}\right) / 2$.

The coherence time and coherence area can be found in a similar way as shown in Section 3. The rough-surfaced retardation plate always has both specular and diffuse component of transmittance. Even the specular component is extremely small when $\sigma_{d}<<\lambda$, but an infinitesimally small specular component, when integrated over infinite limits, will cause the integral to diverge. For this reason, when evaluating the coherence time and the coherence area of the transmitted electric fields by rotating the rough surfaced retardation plate, we must subtract the "DC" component of transmittance, which represents the specular component and make use of $\mu^{2}$ given in Eq. (10) for normalization. Thus the coherence time can be found as

$$
\tau_{c}=\int_{-\infty}^{\infty}\left(\mu^{t}\right)^{2} d \tau,
$$

While this integral does not seem to be expressible in terms of tabulated function, nonetheless it can be evaluated numerically with the results shown in Fig. 8(a), where $\tau_{0}$ is the normalization factor proportional to the time it takes the depolarizer to move a distance of the correlation radius $r_{d}$. As can be seen from the plot, as the standard deviation $\sigma_{d}$ of the thickness of birefringent plate increases, the coherence time falls to smaller and smaller fractions of the time $\tau_{0}$. Note the well-known relation between coherence time and spectral line width $\Delta \gamma \tau_{c}=1$. With a decrease of coherence time for a rotating depolarizer due to an increase of surface roughness for the birefringent material, the spectral distribution of the incident beam is increased. In the same manner, the coherence area can be evaluated with the same result given before (See. Eq. 9 and Fig. 3(a)). Note that the result of spatial coherence is entirely independent of the angular velocity of the rotating rough-surfaced retardation plate.

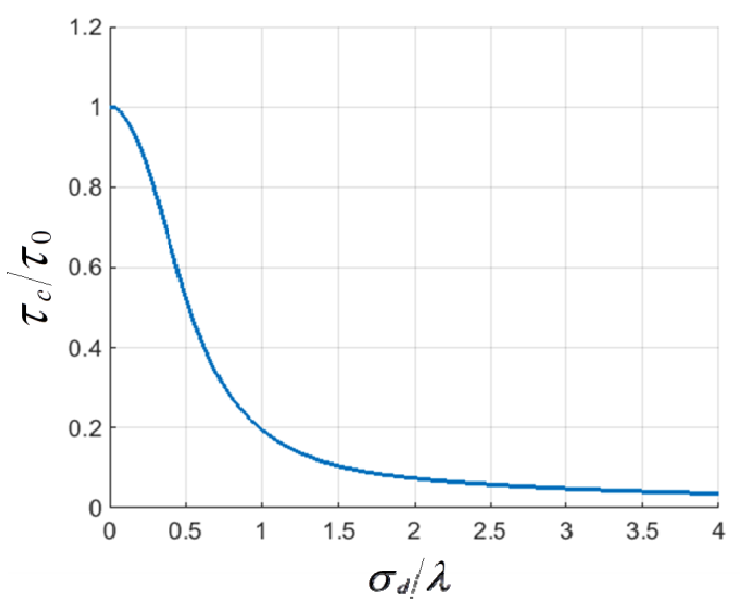

(a)

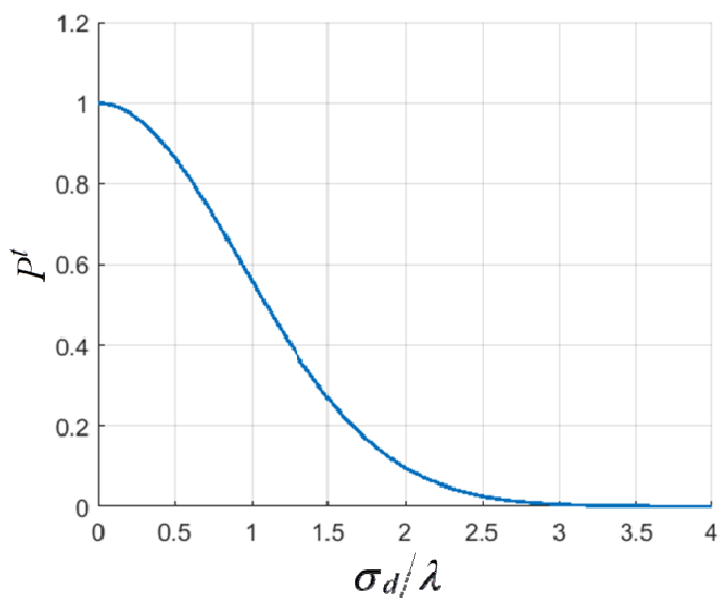

(b)

Fig. 8. The normalized coherence time vs. the normalized standard deviation of thickness (a). The degree of polarization vs. the normalized standard deviation of thickness (b). 
Another significant quantity to investigate is the degree of polarization for the modulated scattered electric field. After taking $\Delta r=0$ and $\tau=0$, we substitute to the mutual coherence tensor of the transmission beam and derive the degree of polarization for the transmitted light through a rotating depolarizer. Figure 8(b) shows the degree of polarization which decreases as the standard deviation of the surface thickness increases. As an example, when the standard deviation of surface thickness is around 3 times the wavelength of the incident light, i.e. $\sigma_{d} / \lambda \approx 3$, the degree of polarization approaches to zero and the transmitted light can be reasonably considered as unpolarized light. From Fig. 3(a) and Fig. 8(a), we note that the coherence area is also close to zero and the corresponding coherence time still reduces to a finite value without becoming zero. Therefore, the beam passing through the rotating rough-surfaced retardation plate can serve as an ideal light source as a quasi-monochromatic, spatially incoherent, unpolarized light source, which has been used for coherence tensor holography for coherence and polarization synthesis.

\section{CONCLUSIONS}

In summary, we reviewed our recent work on the generation and spatio-temporal evolution of polarization speckle generated by a birefringent material with a rough surface. After giving an intuitive explanation of the cause of polarization speckle by vector random walks of polarization phasors, we reviewed the mechanism for the generation of polarization speckle from the rough-surfaced retardation plate and investigated the relationship between the statistical properties of the scattered light and the micro-structure of the anisotropic media. The coherence and polarization properties of the stochastic electric field in the far field after propagation has been studied with respect to its spatial structure and evolution. Furthermore, the dynamic properties of polarization speckle by a rotating rough-surfaced retardation plate have also been examined to explore the simultaneous reduction of coherence and polarization of the scattered light.

\section{REFERENCES}

[1] Dainty, J. C., Ed., [Laser Speckle and Related Phenomena], Springer-Verlag (1984).

[2] Goodman, J. W., [Statistical Optics], Wiley-Interscience, New York (2000).

[3] Goodman, J. W., [Speckle phenomena in Optics: Theory and Applications], Roberts-Company, Colorado (2006).

[4] Wolf, E., [Introduction to the theory of coherence and polarization of light], Cambridge University Press, New York (2007).]

[5] Zhang, S., Takeda, M., and Wang, W., "Statistics of spatial derivatives of Stokes parameters for isotropic random polarization field," JOSA A27, 1999-2003 (2010).

[6] Ma, N., Hanson, S. G., Takeda, M., and Wang, W., "Coherence and polarization of polarization speckle generated by arough-surfaced retardation plate depolarizer," JOSA A 322346-2352 (2015).

[7] Ritter, J., Ma. N., Osten, W., Takeda, M., and Wang, W., "Surface polarization scattering generated from birefringent material with rough surface," Proc. SPIE.10694, 106940J (2018).

[8] Hecht, E., [Optics] Addison-Wesley, (2015).

[9] Wang, W., Matsuda, A., Hanson, S. G., Takeda, M., "The spatial degree of polarization and the first-order statistical properties of polarization speckle," Proc. SPIE 7063, 70630B (2008).

[10] Takeda, M., Wang. W., Naik, D. N. and Singh. R. K., "Spatial statistical optics and spatial correlation holography: a review," Opt. Rev. 21, 849-861 (2014).

[11] Siegman, A. E., [Lasers] University Science, 1986.

[12] Yura, H. T. and Hanson, S. G. "Optical beam wave propagation through complex optical systems," JOSA. A 4, 1931-1948 (1987). 